\title{
A SIMPLE STOCHASTIC KINETIC TRANSPORT MODEL
}

\author{
MICHEL DEKKING *** AND \\ DERONG KONG, ${ }^{* * * *}$ Delft University of Technology
}

\begin{abstract}
We introduce a discrete-time microscopic single-particle model for kinetic transport. The kinetics are modeled by a two-state Markov chain, and the transport is modeled by deterministic advection plus a random space step. The position of the particle after $n$ time steps is given by a random sum of space steps, where the size of the sum is given by a Markov binomial distribution (MBD). We prove that by letting the length of the time steps and the intensity of the switching between states tend to 0 linearly, we obtain a random variable $S(t)$, which is closely connected to a well-known (deterministic) partial differential equation (PDE), reactive transport model from the civil engineering literature. Our model explains (via bimodality of the MBD) the double peaking behavior of the concentration of the free part of solutes in the PDE model. Moreover, we show for instantaneous injection of the solute that the partial densities of the free and adsorbed parts of the solute at time $t$ do exist, and satisfy the PDEs.
\end{abstract}

Keywords: Markov binomial distribution; reactive transport; kinetic adsorption; solute transport; multimodality; double peak

2010 Mathematics Subject Classification: Primary 60J20

Secondary $60 \mathrm{~J} 10$

\section{Introduction}

We consider a mathematical model for the displacement of a solute through a medium which apart from a constant flow (advection) and a dispersion (diffusion) interacts with the medium by intermittent adsorption (the kinetics). Our goal is to connect a stochastic single-particle model to the well-known deterministic model which describes this process by a pair of partial differential equations (PDEs). In Section 2 we introduce the deterministic reactive transport model (as in, e.g. [9]) characterized by a pair of PDEs. In Section 3 we give our simple discretetime microscopic single-particle stochastic reactive transport model. In Section 4 we calculate the probability generating functions of the Markov binomial distribution which is described in Section 3. These are helpful to consider the convergence of our simple discrete-time stochastic model by letting the time step go to 0. This will be discussed in Section 5. In Section 6 we show for instantaneous injection of the solute that the partial probability densities of the free and adsorbed parts of the solute do satisfy the PDEs defined in Section 2. In Section 7 we compute the means and variances of our stochastic reactive transport model. Actually, our formula fills a gap in [9]: since the authors erroneously stated that the variances are linear in

Received 16 June 2011; revision received 21 January 2012.

* Postal address: 3TU Applied Mathematics Institute, Delft University of Technology, Faculty EWI, PO Box 5031, 2600 GA Delft, The Netherlands.

** Email address: f.m.dekking@tudelft.nl

*** Email address: d.kong@tudelft.nl 
the initial distribution, they gave only the result for two initial distributions. In Section 8 we study the probability density function of our stochastic reactive transport model. This gives us a new and more precise point of view at the double peaking behavior in the concentration of the free part of the solute discussed by Michalak and Kitanidis [9].

\section{The PDE reactive transport model}

We briefly describe the model used by Michalak and Kitanidis [9] (see [8] for a more extensive treatment). Given is a solute that has an adsorbed part that does not move, and a free part that moves in the $x$-direction by advection and dispersion. Let $C_{\mathrm{F}}(t, x)$ and $C_{\mathrm{A}}(t, x)$ respectively denote the concentration functions of the free and the adsorbed parts of the solute at time $t$ at position $x$. By applying mass conservation and Fick's law, we can establish the following pair of differential equations:

$$
\begin{gathered}
\frac{\partial C_{\mathrm{F}}(t, x)}{\partial t}+\frac{\partial C_{\mathrm{A}}(t, x)}{\partial t}=D \frac{\partial^{2} C_{\mathrm{F}}(t, x)}{\partial x^{2}}-v \frac{\partial C_{\mathrm{F}}(t, x)}{\partial x}, \\
\frac{\partial C_{\mathrm{A}}(t, x)}{\partial t}=-\mu C_{\mathrm{A}}(t, x)+\lambda C_{\mathrm{F}}(t, x) .
\end{gathered}
$$

Here $D$ is called the dispersion coefficient and $v$ the advection velocity. The parameters $\lambda$ and $\mu$ respectively denote the rates of changes from free to adsorbed and from adsorbed to free. The initial and boundary conditions are given by

$$
C_{\tau}(0, x)=v_{\tau} \delta(x), \quad \lim _{x \rightarrow \infty} C_{\tau}(t, x)=\lim _{x \rightarrow \infty} \frac{\partial C_{\tau}(t, x)}{\partial x}=0 \quad \text { for } t \geq 0, \tau \in\{\mathrm{F}, \mathrm{A}\},
$$

where $\left(v_{\mathrm{F}}, v_{\mathrm{A}}\right)$ is a probability vector and $\delta$ the Dirac delta function.

\section{A simple stochastic reactive transport model}

We describe the behavior of a single particle in the solute. Time $t$ is discretized by choosing some $n$, and dividing $[0, t]$ into $n$ intervals of the same length: $\Delta t=t / n$. We suppose in such an interval of length $\Delta t$ that the particle can be in only one of two states, 'free' or 'adsorbed', which we code by the letters $\mathrm{F}$ and $\mathrm{A}$. The particle can move only when it is 'free', and in this case its displacement has two components, dispersion and advection. Let $X_{k}, k \geq 1$, be the displacement of the particle due to the dispersion the $k$ th time that it is 'free'. We model the $X_{k}$ as independent, identically distributed random variables satisfying

$$
\mathrm{E}_{v}\left[X_{k}\right]=0, \quad \mathrm{E}_{\nu}\left[X_{k}^{2}\right]=2 D \Delta t, \quad \text { and } \quad \mathrm{E}_{\nu}\left[X_{k}^{3}\right]=o(\Delta t) \quad \text { as } \Delta t \downarrow 0,
$$

where $D>0$ and $v=\left(v_{\mathrm{F}}, v_{\mathrm{A}}\right)$ is the initial distribution describing the state of the particle at time 0 . When the particle is free during the interval $[(k-1) \Delta t, k \Delta t]$ for some $k$, the displacement due to advection is given by $v \Delta t$ with $v$ the (deterministic) advection velocity.

In order to model the kinetics, let $\left\{Y_{k}, k \geq 1\right\}$ be a process taking values in $\{\mathrm{F}, \mathrm{A}\}$ (we will make a choice for $\left\{Y_{k}\right\}$ below), and let

$$
K_{n}=\sum_{k=1}^{n} \mathbf{1}_{\left\{Y_{k}=\mathrm{F}\right\}}
$$

be the occupation time of the process $\left\{Y_{k}\right\}$ in state $\mathrm{F}$ up to time $n$. 
Now let $S_{n}(t)$ be the position of the particle at time $t=n \Delta t$, where $S_{n}(0)=0$. Then by the above we can write $S_{n}(t)$ as

$$
S_{n}(t)=\sum_{k=1}^{K_{n}}\left(X_{k}+v \Delta t\right) .
$$

Here we assume that $K_{n}$ is independent of the dispersion $X_{k}, k=1, \ldots, K_{n}$.

We want to compare our stochastic model with the PDE model of Michalak and Kitanidis from Section 2. Since these authors considered the solute with given states ('free' or 'adsorbed') at time $t$, we need to consider the conditional random variables $S_{n}^{\mathrm{F}}(t)$ and $S_{n}^{\mathrm{A}}(t)$, i.e. the position of the particle at time $t$ given that it is 'free' and 'adsorbed', respectively, at time $t=n \Delta t$. Let $K_{n}^{\tau}$ be the random variable $K_{n}$ conditioned on $Y_{n}=\tau$ with $\tau \in\{\mathrm{F}, \mathrm{A}\}$, i.e. $K_{n}^{\tau}$ counts the number of intervals $[(k-1) \Delta t, k \Delta t], 1 \leq k \leq n$, where the particle is free, conditioned on the particle being in state $\tau$ in $[t-\Delta t, t]$. Then $S_{n}^{\tau}(t)$ can be written as

$$
S_{n}^{\tau}(t)=\sum_{k=1}^{K_{n}^{\tau}}\left(X_{k}+v \Delta t\right) .
$$

The distributions of $K_{n}$ and $K_{n}^{\tau}$ are determined by the process $\left\{Y_{k}\right\}$. We take for $\left\{Y_{k}, k \geq 1\right\}$ a Markov chain on the two states $\{\mathrm{F}, \mathrm{A}\}$ with initial distribution $v=\left(v_{\mathrm{F}}, v_{\mathrm{A}}\right)$ and transition matrix

$$
P=\left[\begin{array}{ll}
P(\mathrm{~F}, \mathrm{~F}) & P(\mathrm{~F}, \mathrm{~A}) \\
P(\mathrm{~A}, \mathrm{~F}) & P(\mathrm{~A}, \mathrm{~A})
\end{array}\right]=\left[\begin{array}{cc}
1-a & a \\
b & 1-b
\end{array}\right],
$$

where we assume that $0<a, b<1$. The distribution of $K_{n}$ is then well known, and is called a Markov binomial distribution (MBD) (see, e.g. [4] and [10]).

Clearly, the stationary distribution $\left(\pi_{\mathrm{F}}, \pi_{\mathrm{A}}\right)$ of the Markov chain $\left\{Y_{k}, k \geq 1\right\}$ is given by $\pi_{\mathrm{F}}=b /(a+b)$ and $\pi_{\mathrm{A}}=a /(a+b)$. It is useful to consider the excentricities $\varepsilon_{\mathrm{F}}$ and $\varepsilon_{\mathrm{A}}$ of an initial distribution $v$ given by

$$
\varepsilon_{\tau}:=\varepsilon_{\tau}(\nu)=1-\frac{\nu_{\tau}}{\pi_{\tau}} \quad \text { for } \tau \in\{\mathrm{F}, \mathrm{A}\} .
$$

We can then write $\mathrm{P}_{v}\left(Y_{k}=\tau\right)=\pi_{\tau}\left(1-\varepsilon_{\tau} \gamma^{k-1}\right)$ for $k \geq 1$, where $\gamma=1-a-b$ is the smallest eigenvalue of $P$ (see also [4] for the computations).

\section{Probability generating functions of $K_{n}, K_{n}^{\mathrm{F}}$, and $K_{n}^{\mathrm{A}}$}

Given $n \geq 1$, let $f_{n}$ be the probability mass function of $K_{n}$, i.e.

$$
f_{n}(j)=\mathrm{P}_{v}\left(K_{n}=j\right) .
$$

In particular, $f_{n}(j)=0$ if $j<0$ or $j>n$. Straightforward computations as in [4] or [12] yield

$$
f_{n+2}(j+1)=(1-b) f_{n+1}(j+1)+(1-a) f_{n+1}(j)-(1-a-b) f_{n}(j)
$$

with initial conditions

$$
\begin{aligned}
& f_{1}(0)=v_{\mathrm{A}}, \quad f_{1}(1)=v_{\mathrm{F}}, \\
& f_{2}(0)=v_{\mathrm{A}}(1-b), \quad f_{2}(1)=v_{\mathrm{A}} b+v_{\mathrm{F}} a, \quad f_{2}(2)=v_{\mathrm{F}}(1-a) \text {. }
\end{aligned}
$$


Let $G_{n}$ be the probability generating function of $K_{n}$, i.e.

$$
G_{n}(s)=\mathrm{E}_{v}\left[s^{K_{n}}\right]=\sum_{j=0}^{n} f_{n}(j) s^{j} .
$$

It follows from the above recursion equation for $f_{n}$ that

$$
G_{n+2}(s)=((1-a) s+(1-b)) G_{n+1}(s)-(1-a-b) s G_{n}(s)
$$

with initial conditions

$$
G_{1}(s)=v_{\mathrm{A}}+v_{\mathrm{F}} s, \quad G_{2}(s)=v_{\mathrm{A}}(1-b)+\left(v_{\mathrm{A}} b+v_{\mathrm{F}} a\right) s+v_{\mathrm{F}}(1-a) s^{2} .
$$

By solving the difference equation of $G_{n}$ with these initial conditions we obtain the probability generating function of $K_{n}$ (see also [12]):

$$
G_{n}(s)=g_{n}\left(\alpha_{1}(s), \alpha_{2}(s)\right)+g_{n}\left(\alpha_{2}(s), \alpha_{1}(s)\right) .
$$

Here

$$
g_{n}(x, y)=\frac{v_{\mathrm{A}}(1-y+b(s-1))+v_{\mathrm{F}} s(a-y+s(1-a))}{x-y} x^{n-1},
$$

and

$$
\begin{aligned}
& \alpha_{1}(s)=\frac{1}{2}\left((1-a) s+(1-b)+\sqrt{((1-a) s-(1-b))^{2}+4 a b s}\right), \\
& \alpha_{2}(s)=\frac{1}{2}\left((1-a) s+(1-b)-\sqrt{((1-a) s-(1-b))^{2}+4 a b s}\right) .
\end{aligned}
$$

We now consider the probability generating function of $K_{n}^{\tau}$ for $\tau \in\{\mathrm{F}, \mathrm{A}\}$. Given $n \geq 1$, let $f_{n}^{\tau}$ be the probability mass function of $K_{n}^{\tau}$, i.e.

$$
f_{n}^{\tau}(j)=\mathrm{P}_{v}\left(K_{n}^{\tau}=j\right)=\mathrm{P}_{\nu}\left(K_{n}=j \mid Y_{n}=\tau\right) .
$$

In order to deal with $f_{n}^{\tau}$, it is simpler to deal with the partial probability mass functions

$$
\hat{f}_{n}^{\tau}(j)=\mathrm{P}_{v}\left(K_{n}=j, Y_{n}=\tau\right)=f_{n}^{\tau}(j) \mathrm{P}_{v}\left(Y_{n}=\tau\right),
$$

since these satisfy the same recursion equation as $f_{n}$. Therefore, $G_{n}^{\tau}$, the probability generating function of $K_{n}^{\tau}$, can be obtained similarly to $G_{n}$ by using the appropriate initial conditions (see also [12]). We find that

$$
G_{n}^{\tau}(s)=\sum_{j=1}^{n} f_{n}^{\tau}(j) s^{j}=g_{n}^{\tau}\left(\alpha_{1}(s), \alpha_{2}(s)\right)+g_{n}^{\tau}\left(\alpha_{2}(s), \alpha_{1}(s)\right) \quad \text { for } \tau \in\{\mathrm{F}, \mathrm{A}\},
$$

where

$$
\begin{aligned}
& g_{n}^{\mathrm{F}}(x, y)=\frac{v_{\mathrm{A}} b-v_{\mathrm{F}} y+v_{\mathrm{F}}(1-a) s}{(x-y) \pi_{\mathrm{F}}\left(1-\varepsilon_{\mathrm{F}} \gamma^{n-1}\right)} s x^{n-1}, \\
& g_{n}^{\mathrm{A}}(x, y)=\frac{v_{\mathrm{A}}(1-b)+v_{\mathrm{F}} a s-v_{\mathrm{A}} y}{(x-y) \pi_{\mathrm{A}}\left(1-\varepsilon_{\mathrm{A}} \gamma^{n-1}\right)} x^{n-1} .
\end{aligned}
$$




\section{Towards continuous time}

To get closer to the PDE model in Section 2, we have to fix $t=n \Delta t>0$ and then let the time step $\Delta t$ tend to 0 , or, equivalently, let $n \rightarrow \infty$. We consider the rates of changes $\lambda$ and $\mu$ from Section 2. Since the probability that a particle changes its state is proportional to the length of the time step $\Delta t$ (if $\Delta t$ is small), we should put

$$
a=\lambda \Delta t=\frac{\lambda t}{n}, \quad b=\mu \Delta t=\frac{\mu t}{n}
$$

in the transition matrix $P$ in Section 3. Consider the characteristic function of $S_{n}(t)$,

$$
\varphi_{t, n}(u)=\mathrm{E}_{\nu}\left[\mathrm{e}^{\mathrm{i} u S_{n}(t)}\right]=\mathrm{E}_{v}\left[\exp \left(\mathrm{i} u \sum_{k=1}^{K_{n}}\left(X_{k}+v \Delta t\right)\right)\right]=G_{n}\left(\mathrm{E}_{v}\left[\mathrm{e}^{\mathrm{i} u\left(X_{1}+v \Delta t\right)}\right]\right),
$$

where $G_{n}$ is the generating function of $K_{n}$ given in (4.1). Since $t$ is always assumed to be fixed, we obtain, by (3.1) (cf. [2]),

$$
\begin{aligned}
\mathrm{E}_{v}\left[\mathrm{e}^{\mathrm{i} u\left(X_{1}+v \Delta t\right)}\right] & =1+\mathrm{i} u \mathrm{E}_{v}\left[X_{1}+v \Delta t\right]-\frac{u^{2}}{2} \mathrm{E}_{v}\left[\left(X_{1}+v \Delta t\right)^{2}\right]+o\left(\frac{1}{n}\right) \\
& =1+\frac{t u(\mathrm{i} v-D u)}{n}+o\left(\frac{1}{n}\right)
\end{aligned}
$$

Substituting (5.1) and (5.2) into (4.2), and letting $n$ go to $\infty$, we obtain

$$
\lim _{n \rightarrow \infty} \alpha_{1}\left(\mathrm{E}_{v}\left[\mathrm{e}^{\mathrm{i} u\left(X_{1}+v \Delta t\right)}\right]\right)^{n}=\mathrm{e}^{\theta_{1}(u) t}, \quad \lim _{n \rightarrow \infty} \alpha_{2}\left(\mathrm{E}_{\nu}\left[\mathrm{e}^{\mathrm{i} u\left(X_{1}+v \Delta t\right)}\right]\right)^{n}=\mathrm{e}^{\theta_{2}(u) t},
$$

where

$$
\begin{aligned}
& \theta_{1}(u):=\frac{1}{2}\left(-D u^{2}+\mathrm{i} v u-\lambda-\mu+\sqrt{\left(D u^{2}-\mathrm{i} v u+\lambda-\mu\right)^{2}+4 \lambda \mu}\right), \\
& \theta_{2}(u):=\frac{1}{2}\left(-D u^{2}+\mathrm{i} v u-\lambda-\mu-\sqrt{\left(D u^{2}-\mathrm{i} v u+\lambda-\mu\right)^{2}+4 \lambda \mu}\right) .
\end{aligned}
$$

Here we chose the complex square root of $\left(D u^{2}-\mathrm{i} v u+\lambda-\mu\right)^{2}+4 \lambda \mu$ with positive real part. So, by substituting (5.1) and (5.2) into (4.1) we find that the limit of the characteristic functions $\varphi_{t, n}$ of $S_{n}(t)$ is a function $\varphi_{t}$ given by

$$
\varphi_{t}(u)=\psi_{t}\left(\theta_{1}(u), \theta_{2}(u)\right)+\psi_{t}\left(\theta_{2}(u), \theta_{1}(u)\right)
$$

where

$$
\psi_{t}(x, y)=\frac{\nu_{\mathrm{F}}(x+\lambda+\mu)-v_{\mathrm{A}} y}{x-y} \mathrm{e}^{x t} .
$$

Since $\varphi_{t}$ is continuous at $u=0$, there exists a random variable, which we call $S(t)$, such that, as $n \rightarrow \infty$,

$$
S_{n}(t) \rightarrow S(t) \quad \text { in distribution. }
$$

Similarly, for $S_{n}^{\tau}(t), \tau \in\{\mathrm{F}, \mathrm{A}\}$, we consider its characteristic function

$$
\varphi_{t, n}^{\tau}(u)=\mathrm{E}_{v}\left[\mathrm{e}^{\mathrm{i} u S_{n}^{\tau}(t)}\right]=G_{n}^{\tau}\left(\mathrm{E}_{v}\left[\mathrm{e}^{\mathrm{i} u\left(X_{1}+v \Delta t\right)}\right]\right),
$$


where $G_{n}^{\tau}$ is the probability generating function of $K_{n}^{\tau}$ given in (4.3). Substituting (5.1) and (5.2) into (4.3), and letting $n$ go to $\infty$, we find that the limit of the characteristic functions $\varphi_{t, n}^{\tau}$ of $S_{n}^{\tau}(t)$ is a function $\varphi_{t}^{\tau}$ given by

$$
\varphi_{t}^{\tau}(u)=\psi_{t}^{\tau}\left(\theta_{1}(u), \theta_{2}(u)\right)+\psi_{t}^{\tau}\left(\theta_{2}(u), \theta_{1}(u)\right) \quad \text { for } \tau \in\{\mathrm{F}, \mathrm{A}\},
$$

where

$$
\psi_{t}^{\mathrm{F}}(x, y)=\frac{v_{\mathrm{F}} x+\mu}{(x-y) \pi_{\mathrm{F}}\left(1-\varepsilon_{\mathrm{F}} \mathcal{A}(t)\right)} \mathrm{e}^{x t}, \quad \psi_{t}^{\mathrm{A}}(x, y)=\frac{v_{\mathrm{F}} \lambda-v_{\mathrm{A}}(y+\mu)}{(x-y) \pi_{\mathrm{A}}\left(1-\varepsilon_{\mathrm{A}} \mathcal{A}(t)\right)} \mathrm{e}^{x t},
$$

with $\mathcal{A}(t)=\exp (-(\lambda+\mu) t)$. Here we point out that the stationary distribution $\left(\pi_{\mathrm{F}}, \pi_{\mathrm{A}}\right)$ and the excentricities $\varepsilon_{\mathrm{F}}$ and $\varepsilon_{\mathrm{A}}$ do not depend on the time step $\Delta t$. Again, there exists a random variable, which we call $S^{\tau}(t)$, such that, as $n \rightarrow \infty$,

$$
S_{n}^{\tau}(t) \rightarrow S^{\tau}(t) \quad \text { in distribution. }
$$

\section{Densities and PDEs}

We will show in this section that, for instantaneous injection of the solute, i.e. with initial distribution $v=(1,0)$, the partial probability density functions $\hat{f}_{S}^{\mathrm{F}}(t, x)$ and $\hat{f}_{S}^{\mathrm{A}}(t, x)$ of $S^{\mathrm{F}}(t)$ and $S^{\mathrm{A}}(t)$ do satisfy the PDEs in (2.1).

Let $f_{S}^{\tau}(t, x)$ denote the probability density function of $S^{\tau}(t)$ for $\tau \in\{\mathrm{F}, \mathrm{A}\}$. Note that the probability of a particle being in state $\tau$ at time $t$ is given by

$$
\lim _{n \rightarrow \infty} \mathrm{P}_{v}\left(Y_{n}=\tau\right)=\lim _{n \rightarrow \infty} \pi_{\tau}\left(1-\varepsilon_{\tau} \gamma^{n-1}\right)=\pi_{\tau}\left(1-\varepsilon_{\tau} \mathcal{A}(t)\right) .
$$

We define the partial probability density functions of $S^{\tau}(t)$ by

$$
\hat{f}_{S}^{\tau}(t, x)=\pi_{\tau}\left(1-\varepsilon_{\tau} \mathcal{A}(t)\right) f_{S}^{\tau}(t, x) .
$$

Lemma 6.1. Let $\theta_{1}(u)$ and $\theta_{2}(u)$ be defined as in (5.3). Then

$$
\lim _{u \rightarrow \infty} \theta_{1}(u) u^{2}=\frac{\lambda \mu}{D}, \quad \lim _{u \rightarrow \infty} \frac{\theta_{2}(u)}{u^{2}}=-D .
$$

Proof. It is straightforward to check these formulae.

Lemma 6.2. The probability density function $f_{S}^{\mathrm{F}}(t, \cdot)$ of $S^{\mathrm{F}}(t)$ can be written as

$$
f_{S}^{\mathrm{F}}(t, x)=\frac{1}{2 \pi} \int \mathrm{e}^{-\mathrm{i} u x} \varphi_{t}^{\mathrm{F}}(u) \mathrm{d} u
$$

where $\varphi_{t}^{\mathrm{F}}$ is the characteristic function of $S^{\mathrm{F}}(t)$ given in (5.5).

Proof. We need to only show that $\varphi_{t}^{\mathrm{F}}$ is integrable. Obviously, $\varphi_{t}^{\mathrm{F}}$ is a continuous function. So it suffices to show that $\int_{|u|>M}\left|\varphi_{t}^{\mathrm{F}}(u)\right| \mathrm{d} u<\infty$ for some $M>0$. From Lemma 6.1 and (5.5), it follows that, for all large $|u|$,

$$
\begin{aligned}
\left|\varphi_{t}^{\mathrm{F}}(u)\right| & \leq\left|\frac{\left(\nu_{\mathrm{F}} \theta_{1}(u)+\mu\right) \mathrm{e}^{\theta_{1}(u) t}}{\left(\theta_{1}(u)-\theta_{2}(u)\right) \pi_{\mathrm{F}}\left(1-\varepsilon_{\mathrm{F}} \mathcal{A}(t)\right)}\right|+\left|\frac{\left(\nu_{\mathrm{F}} \theta_{2}(u)+\mu\right) \mathrm{e}^{\theta_{2}(u) t}}{\left(\theta_{2}(u)-\theta_{1}(u)\right) \pi_{\mathrm{F}}\left(1-\varepsilon_{\mathrm{F}} \mathcal{A}(t)\right)}\right| \\
& \leq \frac{C_{1}}{u^{2}}+C_{2} \mathrm{e}^{-D t u^{2} / 2},
\end{aligned}
$$

where $C_{1}$ and $C_{2}$ are constants independent of $u$. This completes the proof. 
Surprisingly, Lemma 6.2 does not hold for $S^{\mathrm{A}}(t)$, but we still have the following result.

Lemma 6.3. The distribution $\mu_{\mathrm{A}}$ of the random variable $S^{\mathrm{A}}(t)$ can be written as

$$
\mu_{\mathrm{A}}=\kappa \delta_{0}+(1-\kappa) \tilde{\mu}_{\mathrm{A}},
$$

where $\kappa=v_{\mathrm{A}} \mathrm{e}^{-\mu t} /\left(\pi_{\mathrm{A}}\left(1-\varepsilon_{\mathrm{A}} \mathcal{A}(t)\right)\right)$ and $\tilde{\mu}_{\mathrm{A}}$ is the distribution of a continuous random variable having probability density function

$$
f_{S}^{\mathrm{A}}(t, x)=\frac{1}{2 \pi(1-\kappa)} \int \mathrm{e}^{-\mathrm{i} u x}\left(\varphi_{t}^{\mathrm{A}}(u)-\kappa\right) \mathrm{d} u,
$$

with $\varphi_{t}^{\mathrm{A}}$ the characteristic function of $S^{\mathrm{A}}(t)$ defined in (5.5).

Proof. It follows from Lemma 6.1 and (5.5) that, for all large $|u|$,

$$
\left|\varphi_{t}^{\mathrm{A}}(u)-\kappa\right| \leq C_{1} \frac{1}{u^{2}}+C_{2} \mathrm{e}^{-D t u^{2} / 2},
$$

where $C_{1}$ and $C_{2}$ are constants. So the integrand in the lemma is integrable.

Without loss of generality, we may suppose that $v_{\mathrm{A}}>0$, so $\kappa>0$. Using the above inequality, we obtain

$$
\lim _{T \rightarrow \infty}\left|\frac{1}{2 T} \int_{-T}^{T} \varphi_{t}^{\mathrm{A}}(u) \mathrm{d} u-\kappa\right|=\lim _{T \rightarrow \infty} \frac{1}{2 T}\left|\int_{-T}^{T}\left(\varphi_{t}^{\mathrm{A}}(u)-\kappa\right) \mathrm{d} u\right|=0 .
$$

This implies that the point 0 is an atom of $\mu_{\mathrm{A}}$, and that it is unique follows by using the uniform boundedness of $\varphi_{t}^{\mathrm{A}}(u)$ (see [2, p. 306]).

It follows from Lemma 6.3 that $S^{\mathrm{A}}(t)$ is a continuous random variable if and only if $v=$ $(1,0)$, i.e. for instantaneous injection of the solute.

Theorem 6.1. The partial probability density functions $\hat{f}_{S}^{\tau}$ of $S^{\tau}(t)$ for $\tau \in\{\mathrm{F}, \mathrm{A}\}$ satisfy the PDEs in (2.1) for $t>0$, i.e.

$$
\begin{gathered}
\frac{\partial \hat{f}_{S}^{\mathrm{F}}(t, x)}{\partial t}+\frac{\partial \hat{f}_{S}^{\mathrm{A}}(t, x)}{\partial t}=D \frac{\partial^{2} \hat{f}_{S}^{\mathrm{F}}(t, x)}{\partial x^{2}}-v \frac{\partial \hat{f}_{S}^{\mathrm{F}}(t, x)}{\partial x}, \\
\frac{\partial \hat{f}_{S}^{\mathrm{A}}(t, x)}{\partial t}=-\mu \hat{f}_{S}^{\mathrm{A}}(t, x)+\lambda \hat{f}_{S}^{\mathrm{F}}(t, x)
\end{gathered}
$$

with initial and boundary conditions

$$
\begin{gathered}
\hat{f}_{S}^{\mathrm{F}}(0, x):=\delta(x), \quad \hat{f}_{S}^{\mathrm{A}}(0, x):=0, \\
\lim _{x \rightarrow \infty} \hat{f}_{S}^{\tau}(t, x)=\lim _{x \rightarrow \infty} \frac{\partial \hat{f}_{S}^{\tau}(t, x)}{\partial x}=0 \quad \text { for } t \geq 0, \tau \in\{\mathrm{F}, \mathrm{A}\} .
\end{gathered}
$$

Proof. The initial conditions imply that $v=(1,0)$. It follows from Lemmas 6.2 and 6.3 and (6.1) that, for $\tau \in\{\mathrm{F}, \mathrm{A}\}$,

$$
\hat{f}_{S}^{\tau}(t, x)=\frac{1}{2 \pi} \int \mathrm{e}^{-\mathrm{i} u x} \hat{\varphi}_{t}^{\tau}(u) \mathrm{d} u,
$$

where $\hat{\varphi}_{t}^{\tau}(u)=\pi_{\tau}\left(1-\varepsilon_{\tau} \mathcal{A}(t)\right) \varphi_{t}^{\tau}(u)$ with $\varphi_{t}^{\tau}$ the characteristic functions of $S^{\tau}(t)$ given in (5.5). It is easy to see that $\hat{f}_{S}^{\mathrm{F}}$ and $\hat{f}_{S}^{\mathrm{A}}$ satisfy the initial and boundary conditions. 
Using Lemma 6.1, it is not hard to check that the partial derivatives of $\mathrm{e}^{-\mathrm{i} u x} \hat{\varphi}_{t}^{\tau}(u)$ with respect to $x$ and with respect to $t$ are all bounded by a function of the form $C_{1} / u^{2}+C_{2} \mathrm{e}^{-D t u^{2} / 2}$ for large $|u|$, where $C_{1}$ and $C_{2}$ are constants independent of $u$. Thus, we can exchange the integral and differential operators in the PDEs (cf. [5, p. 417]). Hence, to complete the proof, we need to only show that

$$
\begin{aligned}
& \frac{\partial \hat{\varphi}_{t}^{\mathrm{F}}(u)}{\partial t}=-D u^{2} \hat{\varphi}_{t}^{\mathrm{F}}(u)+\mathrm{i} v u \hat{\varphi}_{t}^{\mathrm{F}}(u)-\lambda \hat{\varphi}_{t}^{\mathrm{F}}(u)+\mu \hat{\varphi}_{t}^{\mathrm{A}}(u), \\
& \frac{\partial \hat{\varphi}_{t}^{\mathrm{A}}(u)}{\partial t}=-\mu \hat{\varphi}_{t}^{\mathrm{A}}(u)+\lambda \hat{\varphi}_{t}^{\mathrm{F}}(u) .
\end{aligned}
$$

These can be checked by using $(5.5), v_{\mathrm{A}}=0$, and the easily verified equality $\left(\theta_{1}(u)+\mu\right)\left(\theta_{2}(u)+\right.$ $\mu)=-\lambda \mu$.

We would like to point out that Lindstrom and Narasimhan [8] gave an analytical solution of the PDEs with different initial and boundary conditions by using Laplace and inverse Laplace transforms. Their method can also be used with our initial and boundary conditions to give the same densities as we have obtained via our stochastic model as in Theorem 6.1.

\section{Moments of $S(t), S^{\mathrm{F}}(t)$, and $S^{\mathrm{A}}(t)$}

The mean and variance of $S(t)$ and $S^{\tau}(t), \tau \in\{\mathrm{F}, \mathrm{A}\}$, can be obtained by differentiating their characteristic functions in (5.4) and (5.5), or, more leisurely, by taking the limits of $\mathrm{E}_{v}\left[S_{n}(t)\right], \operatorname{var}_{v}\left(S_{n}(t)\right), \mathrm{E}_{v}\left[S_{n}^{\tau}(t)\right]$, and $\operatorname{var}_{v}\left(S_{n}^{\tau}(t)\right)$, respectively. These are allowed (see, e.g. [2, Theorem 25.12]) by the easily proved uniform integrability of $S_{n}(t),\left(S_{n}(t)\right)^{2}, S_{n}^{\tau}(t)$, and $\left(S_{n}^{\tau}(t)\right)^{2}$, which is implied by the uniform boundedness of $\left(S_{n}(t)\right)^{3}$ and $\left(S_{n}^{\tau}(t)\right)^{3}$.

Since $X_{k}$ is independent of $K_{n}$, from (4) and Proposition 2.1 of [4] together with (3.1), we can determine the first and second moments of $S_{n}(t)$. The following proposition can be obtained by substituting (5.1) into the moments of $S_{n}(t)$ and letting $n \rightarrow \infty$.

Proposition 7.1. The mean and variance of $S(t)$ are given by

$$
\mathrm{E}_{v}[S(t)]=\pi_{\mathrm{F}} v t-\frac{\varepsilon_{\mathrm{F}} \pi_{\mathrm{F}}}{\lambda+\mu} v(1-\mathcal{A}(t))
$$

and

$$
\begin{aligned}
\operatorname{var}_{v}(S(t))= & 2 D \pi_{\mathrm{F}} t-\frac{2 D \varepsilon_{\mathrm{F}} \pi_{\mathrm{F}}}{\lambda+\mu}(1-\mathcal{A}(t))+\frac{2\left(\pi_{\mathrm{A}}+\varepsilon_{\mathrm{F}}\left(\pi_{\mathrm{A}}-\pi_{\mathrm{F}}\right) \mathcal{A}(t)\right)}{\lambda+\mu} \pi_{\mathrm{F}} v^{2} t \\
& +\frac{\varepsilon_{\mathrm{F}}\left(\pi_{\mathrm{F}}-\pi_{\mathrm{A}}\right)-2 \pi_{\mathrm{A}}-\varepsilon_{\mathrm{F}}\left(\pi_{\mathrm{A}}-v_{\mathrm{F}}\right)}{(\lambda+\mu)^{2}} \pi_{\mathrm{F}} v^{2} \\
& +\mathcal{A}(t)\left(2 \frac{\pi_{\mathrm{A}}+\varepsilon_{\mathrm{F}}\left(\pi_{\mathrm{A}}-v_{\mathrm{F}}\right)}{(\lambda+\mu)^{2}}-\mathcal{A}(t) \frac{\pi_{\mathrm{F}} \varepsilon_{\mathrm{F}}^{2}}{(\lambda+\mu)^{2}}\right) \pi_{\mathrm{F}} v^{2} .
\end{aligned}
$$

If we start in the stationary distribution, i.e. $v=\left(\pi_{\mathrm{F}}, \pi_{\mathrm{A}}\right)$, then $\varepsilon_{\mathrm{F}}=\varepsilon_{\mathrm{A}}=0$. Substituting $\pi_{\mathrm{F}}=\mu /(\lambda+\mu), \pi_{\mathrm{A}}=\lambda /(\lambda+\mu)$, and $\mathcal{A}(t)=\exp (-(\lambda+\mu) t)$ into (7.1), we obtain

$$
\operatorname{var}_{\nu}(S(t))=\frac{2 D \mu}{\lambda+\mu} t+\frac{2 \mu \lambda}{(\lambda+\mu)^{3}} v^{2} t-\frac{2 \mu \lambda}{(\lambda+\mu)^{4}} v^{2}(1-\exp (-(\lambda+\mu) t)) .
$$

We then recover a (more general and more detailed) version of the main result of Gut and Ahlberg [6, p. 251]. 
In a similar way as for Proposition 7.1, the mean and variance of $S_{n}^{\mathrm{F}}(t)$ can be obtained by using (5) and Proposition 3.1 of [4] together with (3.1). Substituting (5.1) into the moments of $S_{n}^{\mathrm{F}}(t)$ and letting $n \rightarrow \infty$, we obtain the following result.

Proposition 7.2. The mean and variance of $S^{\mathrm{F}}(t)$ are given by

$$
\mathrm{E}_{v}\left[S^{\mathrm{F}}(t)\right]=\frac{\pi_{\mathrm{F}}-\varepsilon_{\mathrm{F}} \pi_{\mathrm{A}} \mathcal{A}(t)}{1-\varepsilon_{\mathrm{F}} \mathcal{A}(t)} v t+\frac{\left(\pi_{\mathrm{A}}-\varepsilon_{\mathrm{F}} \pi_{\mathrm{F}}\right)(1-\mathcal{A}(t))}{(\lambda+\mu)\left(1-\varepsilon_{\mathrm{F}} \mathcal{A}(t)\right)} v
$$

and

$$
\begin{aligned}
\operatorname{var}_{v}\left(S^{\mathrm{F}}(t)\right)= & \frac{\pi_{\mathrm{F}}-\varepsilon_{\mathrm{F}} \pi_{\mathrm{A}} \mathcal{A}(t)}{1-\varepsilon_{\mathrm{F}} \mathcal{A}(t)} 2 D t+\frac{\pi_{\mathrm{A}}-\varepsilon_{\mathrm{F}} \pi_{\mathrm{F}}}{(\lambda+\mu)\left(1-\varepsilon_{\mathrm{F}} \mathcal{A}(t)\right)} 2 D(1-\mathcal{A}(t)) \\
& +\frac{\pi_{\mathrm{F}}^{2}-\varepsilon_{\mathrm{F}} \pi_{\mathrm{A}}^{2} \mathcal{A}(t)}{1-\varepsilon_{\mathrm{F}} \mathcal{A}(t)} v^{2} t^{2}-2 \frac{\varepsilon_{\mathrm{F}} \pi_{\mathrm{F}}^{2}+\pi_{\mathrm{A}}^{2} \mathcal{A}(t)-2 \pi_{\mathrm{A}} \pi_{\mathrm{F}}\left(1+\varepsilon_{\mathrm{F}} \mathcal{A}(t)\right)}{(\lambda+\mu)\left(1-\varepsilon_{\mathrm{F}} \mathcal{A}(t)\right)} v^{2} t \\
& -\left(\frac{\pi_{\mathrm{F}}-\varepsilon_{\mathrm{F}} \pi_{\mathrm{A}} \mathcal{A}(t)}{1-\varepsilon_{\mathrm{F}} \mathcal{A}(t)} t+\frac{\pi_{\mathrm{A}}-\varepsilon_{\mathrm{F}} \pi_{\mathrm{F}}}{(\lambda+\mu)\left(1-\varepsilon_{\mathrm{F}} \mathcal{A}(t)\right)}(1-\mathcal{A}(t))\right)^{2} v^{2} \\
& +2(1-\mathcal{A}(t)) \frac{\varepsilon_{\mathrm{F}} \pi_{\mathrm{F}}^{2}+\pi_{\mathrm{A}}^{2}-2 \pi_{\mathrm{A}} \pi_{\mathrm{F}}\left(1+\varepsilon_{\mathrm{F}}\right)}{(\lambda+\mu)^{2}\left(1-\varepsilon_{\mathrm{F}} \mathcal{A}(t)\right)} v^{2} .
\end{aligned}
$$

Now, we will use our model to illustrate a mistake made by Michalak and Kitanidis [9]. They did not directly use $\lambda$ and $\mu$, but rather considered a distribution coefficient $K_{d}$ and a mass transfer coefficient $k$, which are given by

$$
\lambda=\beta k=\frac{\rho K_{d}}{\eta} k, \quad \mu=k .
$$

If we let the solute be 'free' at time 0 , i.e. the initial distribution is $v=(1,0)$, then

$$
\varepsilon_{\mathrm{F}}=-\frac{\lambda}{\mu}=-\beta, \quad \pi_{\mathrm{F}}=\frac{\mu}{\lambda+\mu}=\frac{1}{\beta+1}, \quad \pi_{\mathrm{A}}=\frac{\lambda}{\lambda+\mu}=\frac{\beta}{\beta+1} .
$$

Substituting these parameters into (7.2) yields

$$
\begin{aligned}
\operatorname{var}_{\mathrm{F}}\left(S^{\mathrm{F}}(t)\right)= & \frac{t^{2} \mathcal{A}(t) v^{2} \beta(\beta-1)^{2}}{(\beta+1)^{2}(1+\beta \mathcal{A}(t))^{2}}+t\left(\frac{2 D}{\beta+1}+\frac{2 v^{2} \beta}{k(\beta+1)^{3}}\right)+\frac{4 D \beta(1-\mathcal{A}(t))}{k(1+\beta \mathcal{A}(t))(\beta+1)^{2}} \\
& +t \mathcal{A}(t)\left(\frac{4 v^{2} \beta\left(-\beta^{2} \mathcal{A}(t)-\beta^{2}-\beta+1\right)}{k(1+\beta \mathcal{A}(t))^{2}(\beta+1)^{3}}+\frac{2 D(\beta-1)}{(\beta+1)(1+\beta \mathcal{A}(t))}\right) \\
& +\frac{2 v^{2} \beta(1-\mathcal{A}(t))\left(3 \beta^{2} \mathcal{A}(t)-3-\beta(\mathcal{A}(t) \oplus 1)\right)}{k^{2}(1+\beta \mathcal{A}(t))^{2}(\beta+1)^{4}},
\end{aligned}
$$

where $\operatorname{var}_{\mathrm{F}}\left(S^{\mathrm{F}}(t)\right):=\operatorname{var}_{(1,0)}\left(S^{\mathrm{F}}(t)\right)$ and $\mathcal{A}(t)=\exp (-(\lambda+\mu) t)=\exp (-(\beta+1) k t)$. Indeed, this gives the formula in [9, p. 2136] (the ' $\oplus$ ' is ' + ' in [9], but should be ' - ').

Michalak and Kitanidis stated in their paper that $\operatorname{var}_{v}\left(S^{\tau}(t)\right)$ can be obtained by a linear combination of $\operatorname{var}_{\mathrm{F}}\left(S^{\tau}(t)\right)$ and $\operatorname{var}_{\mathrm{A}}\left(S^{\tau}(t)\right.$ ) (i.e. $\operatorname{var}_{v}\left(S^{\tau}(t)\right)$ with initial distributions $v=$ $(1,0)$ and $v=(0,1))$. This is not true, and we provide the correct formula for the free case in Proposition 7.2. The formula for the adsorbed case can be obtained in a similar way. 


\section{Double-peak behavior in reactive transport models}

Double peaks in the 'free' concentration distribution $C_{\mathrm{F}}$ are discussed by Michalak and Kitanidis [9] using simulations. Theorem 6.1 tells us that $C_{\mathrm{F}}(t, \cdot)$ can be seen as the partial probability density function $\hat{f}_{S}^{\mathrm{F}}(t, \cdot)$ of $S^{\mathrm{F}}(t)$ if the initial distribution $v=(1,0)$. We will show in this section how double peaks can also be mathematically explained by means of our stochastic reactive transport model. Let $f_{S_{n}}^{\mathrm{F}}(t, \cdot)$ be the probability density function of $S_{n}^{\mathrm{F}}(t)$ defined in Section 3. We are going to approximate $f_{S}^{\mathrm{F}}(t, \cdot)$ by $f_{S_{n}}^{\mathrm{F}}(t, \cdot)$ since $S_{n}^{\mathrm{F}}(t)$ converges to $S^{\mathrm{F}}(t)$ in distribution.

Michalak and Kitanidis considered Gaussian diffusion, i.e. the $X_{k}$ s are normally distributed random variables with mean 0 and variance $2 D \Delta t$, which satisfy (3.1). So the characteristic function of $S_{n}^{\mathrm{F}}(t)$ can be written as

$$
\varphi_{t, n}^{\mathrm{F}}(u)=\mathrm{E}_{v}\left[\mathrm{e}^{\mathrm{i} u S_{n}^{\mathrm{F}}(t)}\right]=G_{n}^{\mathrm{F}}\left(\mathrm{E}_{v}\left[\mathrm{e}^{\mathrm{i} u\left(X_{1}+v \Delta t\right)}\right]\right)=\sum_{j=0}^{n} f_{n}^{\mathrm{F}}(j) \exp \left(\mathrm{i} v \Delta t j u-D \Delta t j u^{2}\right),
$$

where $f_{n}^{\mathrm{F}}$ is the probability mass function of $K_{n}^{\mathrm{F}}$. Obviously, $\int_{-\infty}^{\infty}\left|\varphi_{t, n}^{\mathrm{F}}(u)\right| \mathrm{d} u<\infty$. Thus, by the inverse Fourier transformation, using the fact that $f_{n}^{\mathrm{F}}(0)=0$, we obtain

$$
f_{S_{n}}^{\mathrm{F}}(t, x)=\frac{1}{2 \pi} \int_{-\infty}^{\infty} \mathrm{e}^{-\mathrm{i} u x} \varphi_{t, n}^{\mathrm{F}}(u) \mathrm{d} u=\sum_{j=1}^{n} \frac{f_{n}^{\mathrm{F}}(j)}{\sqrt{4 \pi j D \Delta t}} \exp \left(-\frac{(x-j v \Delta t)^{2}}{4 j D \Delta t}\right) .
$$

So $S_{n}^{\mathrm{F}}(t)$ is a mixture of Gaussian distributions with mean $j v \Delta t$ and variance $2 j D \Delta t$. Recall from [4] that the probability mass function $f_{n}^{\mathrm{F}}$ of $K_{n}^{\mathrm{F}}$ can be unimodal or bimodal. This property of $K_{n}^{\mathrm{F}}$ gives rise to the same phenomenon for $S_{n}^{\mathrm{F}}(t)$, i.e. one peak or two peaks appear in the probability density function $f_{S_{n}}^{\mathrm{F}}(t, x)$ of $S_{n}^{\mathrm{F}}=S_{n}^{\mathrm{F}}(t)$ for large $n$.

Michalak and Kitanidis focused on the case in which the solute is initially in the free phase and evenly distributed over an interval of length $L$, i.e. the initial conditions of the PDEs in (2.1) are given by

$$
C_{\mathrm{F}}(0, x)=\frac{1}{L} \mathbf{1}_{[0, L]}(x), \quad C_{\mathrm{A}}(0, x)=0 .
$$

So, to make the comparison, we look at the probability density function $f_{\tilde{S}_{n}}^{\mathrm{F}}(t, x)$ of

$$
\tilde{S}_{n}^{\mathrm{F}}(t)=S_{n}^{\mathrm{F}}(t)+U_{L},
$$

where $U_{L}$ is a uniformly distributed random variable over the interval $[0, L]$. Michalak and Kitanidis pointed out that the double peaking behavior of the free concentration distribution is a function of the so-called Damköhler number of the first kind, $\mathrm{Da}_{I}=\mu L R / v$, where $R$ is the dimensionless retardation coefficient. They stated that the timing of its appearance is controlled by the mass transfer rate and the retardation factor, i.e. the dimensionless time $t^{*}=\mu(R-1) t$. The so-called Péclet number $\mathrm{Pe}=v L / D$ is kept constant at a value of 100 . We translate these parameters into our paper as follows:

$$
a=\frac{t^{*}}{n}, \quad b=\frac{t^{*}}{n(R-1)}, \quad D=\frac{v L}{\mathrm{Pe}}, \quad \Delta t=\frac{t}{n}=\frac{t^{*} L R}{n v(R-1) \mathrm{Da}_{I}} .
$$

The left-hand graphs in Figure 1 are a copy of the graphs of the normalized aqueous concentration functions $C_{\mathrm{F}}(t, \cdot) / \max _{x} C_{\mathrm{F}}(t, x)$ (consisting of the free particles) in Michalak 


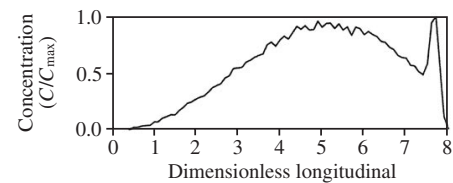

displacement $(\mathrm{xkR} / \mathrm{v})$

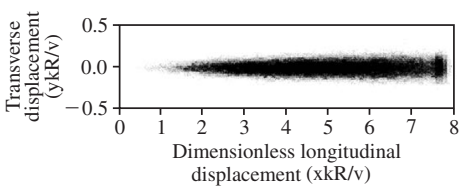

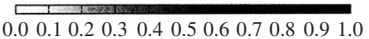

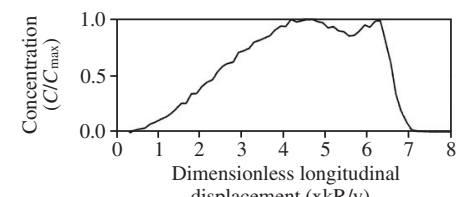

displacement $(\mathrm{xkR} / \mathrm{v})$

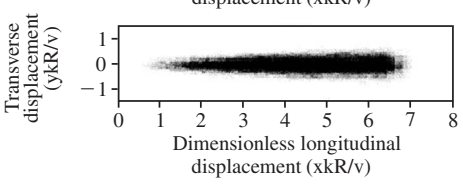

0.00 .10 .20 .30 .40 .50 .60 .70 .80 .91 .0
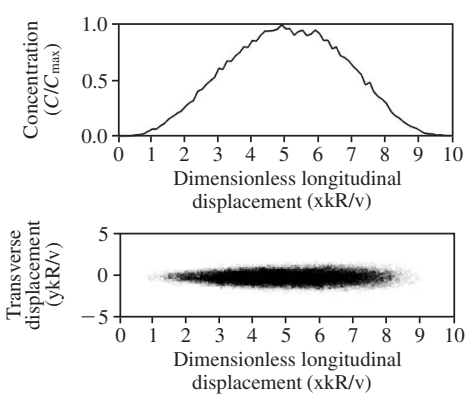

0.00 .10 .20 .30 .40 .50 .60 .70 .80 .91 .0
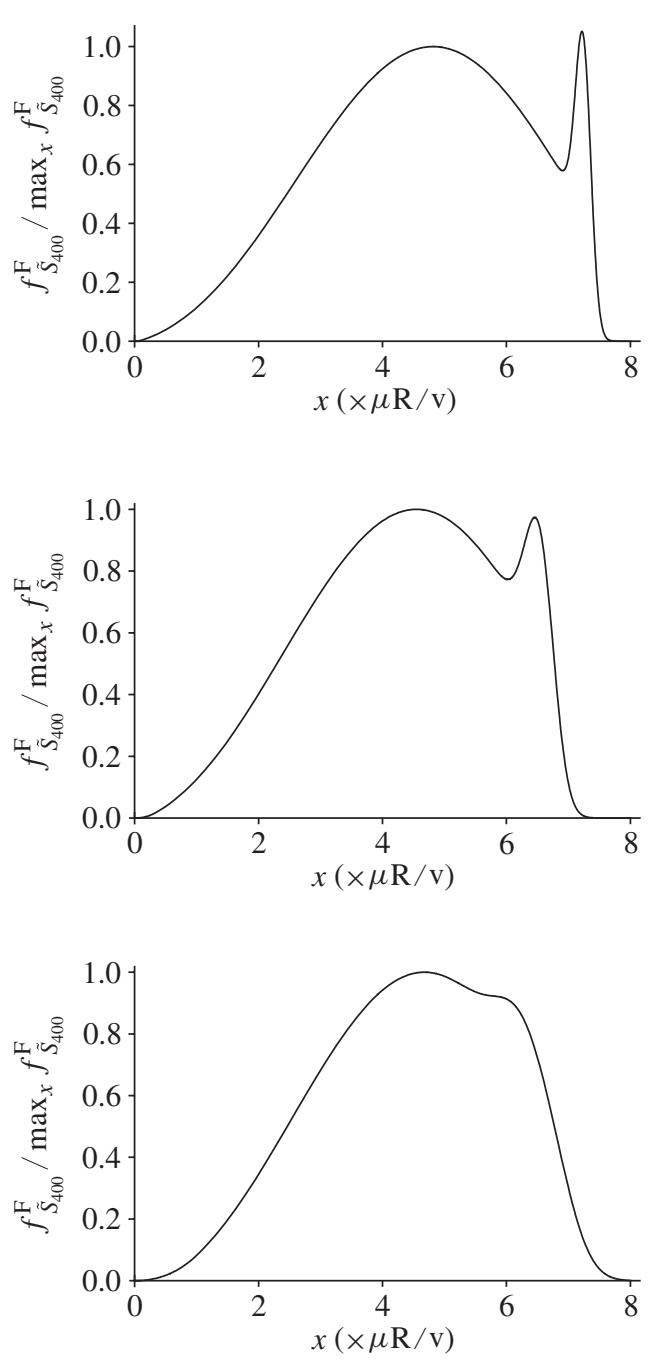

Figure 1: The left-hand graphs are the normalized concentration functions $C_{\mathrm{F}}(t, \cdot) / \max _{x} C_{\mathrm{F}}(t, x)$ copied from Michalak and Kitanidis [9]. The right-hand graphs are the normalized probability density functions $f_{\tilde{S}_{400}}^{\mathrm{F}}(t, \cdot) / \max _{x} f_{\tilde{S}_{400}}^{\mathrm{F}}(t, x)$ given in our paper. All graphs have Pe $=100, v=L=1$, and $R=2$, with $\mathrm{Da}_{I}=0.1$ and $t^{*}=3.6$ (top row), $\mathrm{Da}_{I}=0.33$ and $t^{*}=3.2$ (middle row), and $\mathrm{Da}_{I}=1.0$ and $t^{*}=3.0$ (bottom row).

and Kitanidis [9] using simulations corresponding to different choices of the Damköhler number $\mathrm{Da}_{I}$ and dimensionless time $t^{*}$. The right-hand graphs in Figure 1 are the normalized density functions $f_{\tilde{S}_{400}}^{\mathrm{F}}(t, \cdot) / \max _{x} f_{\tilde{S}_{400}}^{\mathrm{F}}(t, x)$ calculated using (8.1), and the same choices of $\mathrm{Da}_{I}$ and $t^{*}$. The number $n$ is chosen large enough such that $\max (a, b)=\max (\lambda \Delta t, \mu \Delta t) \leq 0.01$. From Figure 1, it is obvious that our model gives a much better view at the double peaking phenomenon.

Moreover, for each $t^{*}$, by a numerical calculation we can obtain upper bounds for $\mathrm{Da}_{I}$ such that double peaks appear (see Table 1). For example, when $t^{*}=2.0$, two peaks occur for 
TABLE 1: $R=2, \mathrm{Pe}=100, v=1, L=1, n=400$.

\begin{tabular}{lccccccccccccc}
\hline$t^{*}$ & 1.5 & 2.0 & 2.5 & 3.0 & 3.5 & 4.0 & 4.5 & 5.0 & 6.0 & 7.0 & 8.0 & 9.0 & 10.0 \\
$\mathrm{Da}_{I}^{\max }$ & 0.12 & 0.43 & 1.45 & 1.42 & 0.73 & 0.45 & 0.30 & 0.21 & 0.11 & 0.07 & 0.04 & 0.02 & 0.02 \\
\hline
\end{tabular}

all $\mathrm{Da}_{I}>0$ until $\mathrm{Da}_{I}=\mathrm{Da}_{I}^{\max }=0.43$. Table 1 suggests that double peaking is pronounced for $2 \leq t^{*} \leq 5$, and almost dies out when $t^{*}<1.5$ or $t^{*}>10$.

\section{Final remarks}

We emphasize that the so-called 'random walk method' or 'particle tracking method' first proposed by Kinzelbach [7] has a relation to our model, but has typically been used as a simulation tool to perform numerical experiments (for a recent example, see [1]). In fact, it was shown in [11] for the first time that if one takes an appropriate limit (in a similar way as in [3]), then the Fokker-Planck equations of an extended version of our simple model to a Markov chain, which also involves discrete steps in space, yield the partial differential equations in (2.1).

Finally, we remark that if we consider a continuous-time model, with displacement modeled by Brownian motion with drift $v$, and kinetics induced by the natural continuous-time $2 \times 2$ Markov chain with rates $\lambda$ and $\mu$, then we can show that the same random variables $S(t), S^{\mathrm{F}}(t)$, and $S^{\mathrm{A}}(t)$ are obtained.

\section{Acknowledgements}

The first author would like to thank J. Bruining, G. Uffink, and C. Kraaikamp for numerous enlightening conversations. The second author was partially supported by the National Natural Science Foundation of China (10971069) and the Shanghai Education Committee Project (11ZZ41).

\section{References}

[1] Benson, D. A. and Meerschaert, M. M. (2009). A simple and efficient random walk solution of multi-rate mobile/immobile mass transport equations. Adv. Water Resources 32, 532-539.

[2] Billingsley, P. (1995). Probability and Measure, 3rd edn. John Wiley, New York.

[3] Dehling, H. G., Hoffmann, A. C. and Stuut, H. W. (2000). Stochastic models for transport in a fluidized bed. SIAM J. Appl. Math. 60, 337-358.

[4] Dekking, M. And Kong, D. (2011). Multimodality of the Markov binomial distribution. J. Appl. Prob. 48, 938-953.

[5] Durrett, R. (2010). Probability: Theory and Examples, 4th edn. Cambridge University Press.

[6] Gut, A. And Ahlberg, P. (1981). On the theory of chromatography based upon renewal theory and a central limit theorem for randomly iterated indexed partial sums of random variables. Chemica Scripta 18, $248-255$.

[7] Kinzelbach, W. (1988). The random walk method in pollutant transport simulation. In Groundwater Flow and Quality Modelling (NATO ASI Ser. C Math. Phys. Sci. 224), pp. 227-245.

[8] Lindstrom, F. T. and Narasimham, M. N. L. (1973). Mathematical theory of a kinetic model for dispersion of previously distributed chemicals in a sorbing porous medium. SIAM J. Appl. Math. 24, 496-510.

[9] MichalaK, A. M. AND Kitanidis, P. K. (2000). Macroscopic behavior and random-walk particle tracking of kinetically sorbing solutes. Water Resources Res. 36, 2133-2146.

[10] Omey, E., Santos, J. And Van Gulck, S. (2008). A Markov-binomial distribution. Appl. Anal. Discrete Math. 2, 38-50.

[11] UfFINK, G. et al. (2012). Understanding the non-Gaussian nature of linear reactive solute transport in 1D and 2D: from particle dynamics to the partial differential equations. Transport Porous Media 91, 547-571.

[12] Viveros, R., Balasubramanian, K. and Balakrishnan, N. (1994). Binomial and negative binomial analogues under correlated Bernoulli trials. Amer. Statist. 48, 243-247. 